\title{
Measuring localized viscoelasticity of the vitreous body using intraocular microprobes
}

\author{
Juho Pokki ${ }^{1}$ (D) Olgaç Ergeneman ${ }^{1} \cdot$ Semih Sevim $^{2} \cdot$ Volker Enzmann $^{3}$ • \\ Hamdi Torun $^{2,4}$ - Bradley J. Nelson ${ }^{1}$
}

Published online: 4 August 2015

(C) Springer Science+Business Media New York 2015

\begin{abstract}
Vitrectomy is a standard ophthalmic procedure to remove the vitreous body from the eye. The biomechanics of the vitreous affects its duration (by changing the removal rate) and the mechanical forces transmitted via the vitreous on the surrounding tissues during the procedure. Biomechanical characterization of the vitreous is essential for optimizing the design and control of instruments that operate within the vitreous for improved precision, safety, and efficacy. The measurements are carried out using a magnetic microprobe inserted into the vitreous, a method known as magnetic microrheology. The location of the probe is tracked by a microscope/camera while magnetic forces are exerted wirelessly by applied magnetic fields. In this work, in vitro artificial vitreous, ex vivo human vitreous and ex vivo porcine vitreous were characterized. In addition, in vivo rabbit measurements were performed using a suturelessly injected probe. Measurements indicate that viscoelasticity parameters of the ex vivo human vitreous are an order of magnitude different from those of the ex vivo porcine vitreous. The in vivo intraoperative measurements show typical viscoelastic behavior of the vitreous with a lower compliance than the ex vivo measurements. The results of the magnetic microrheology
\end{abstract}

Juho Pokki

jpokki@ethz.ch

1 Institute of Robotics and Intelligent Systems, ETH Zurich, Tannenstrasse 3, 8092 Zurich, Switzerland

2 Department of Electrical and Electronics Engineering, Boğaziçi University, Istanbul 34342, Turkey

3 Department of Ophthalmology, Inselspital, University of Bern, Bern 3010, Switzerland

4 Center for Life Sciences and Technologies, Boğaziçi University, Istanbul 34684, Turkey measurements were validated with those obtained by a standard atomic force microscopy (AFM) method and in vitro artificial vitreous. This method allows minimally-invasive characterization of localized mechanical properties of the vitreous in vitro, ex vivo, and in vivo. A better understanding of the characteristics of the vitreous can lead to improvements in treatments concerning vitreal manipulation such as vitrectomy.

Keywords Vitreous body · Microprobe - Viscoelasticity · Magnetic microrheology $\cdot$ Atomic force microscopy . Vitrectomy

\section{Introduction}

The vitreous is a functional gel-like hydrated body filling the inner cavity of the eye. It helps to protect and support the surrounding eye structures. Although the vitreous is 98 $99 \%$ water, it exhibits complex viscoelastic properties mainly due to the presence of hyaluronan (HA) and a network of heterotypic collagen fibrils (Bishop 2000; Sharif-Kashani et al. 2011). The voids of this network are filled with HA that serves to stabilize the vitreous with the help of less abundant molecules (proteoglycans, glycoproteins) (Ponsioen et al. 2010).

The properties of the vitreous body are altered during aging (Sebag 1987; Los et al. 2003). Liquefaction is a typical change, however, the mechanisms leading to it are still not fully understood. Fluid-filled cavities (lacunae) are gradually formed in the proximity of the vitreous core. The distribution of collagen, HA, and other macromolecules change by age or from disease (Nguyen et al. 2005; Sebag et al. 1992, 1993, 1994, 1999). For example, collagen fibrils can collapse and form bundles (Los et al. 2003; Filas et al. 2014). A detachment 
of the vitreous from the retina may occur, which can lead to vision pathologies in more severe conditions (e.g., vitreomacular traction, macular pucker, or macular hole). These changes alter drastically the spatially-varying viscoelastic properties that depend on macromolecular organization (Bishop 2000; Sharif-Kashani et al. 2011; Lee et al. 1992, 1994a, b). Viscoelastic properties also play an important role in the onset of several vitreous-related complications (e.g., glaucoma, vitreous hemorrhage). Elucidating biomechanical properties of the vitreous contributes to a better understanding of the pathophysiological events and may lead to improved diagnosis (Sebag et al. 1999; Ansari et al. 2001).

A conventional method of intervention in the vitreous is pars plana vitrectomy. Viscoelastic interaction during the vitrectomy determines the dynamics of the instruments in the vitreous, although little quantitative data has been reported characterizing interaction forces (Sharif-Kashani et al. 2013). A method to characterize in vivo viscoelasticity prior to vitrectomy or other ophthalmic procedures could lead to safer and more efficient procedures (e.g., in vitrectomy, finding optimal removal rate could shorten duration). The knowledge on viscoelastic properties can be used to set control parameters of ophthalmic instruments, such as force, position, and velocity. Vitreous characterization helps limiting the force and strain levels, for example, to minimize risk of vitreoretinal traction (Schneider and Johnson 2011). Characterization of the viscoelastic properties of the vitreous is also necessary for optimizing the design of ophthalmic instruments, including their shape, size, and surface properties.

The viscoelasticity of the vitreous has previously been studied using methods involving dissection (Zimberlin et al. 2010) or removal of the vitreous (Sharif-Kashani et al. 2011) in addition to the probeless techniques including dynamic light scattering (Sebag et al. 2007). These procedures can alter the internal structure of the vitreous and, hence, its viscoelastic properties. Viscoelasticity can be characterized minimally invasively inside an eye by microscopic probes (i.e., active microrheology) as in the paper of Pokki et al. (2012). Microrheology offers higher spatial resolution and dynamic range than traditional rheology. However, measuring elasticity and viscosity from viscoelastic biofluids such as the vitreous, is challenging. Creep experiments in microrheology have been found to be the most direct way to measure their elasticity (Ferry 1980).

In this work, an experimental microrheology system based on manipulation of a magnetic microprobe was developed. The system capable of localized measurements inside an intact eye was employed to characterize viscoelastic properties of the human vitreous. It has a workspace the size of a human eye. Measurements (i.e., creep experiments) in synthesized artificial vitreous, ex vivo human vitreous (age: 75 years) and ex vivo porcine vitreous were performed. In addition, in vivo measurements in a rabbit eye were performed with the same system. The results of the magnetic microrheology measurements were compared with those obtained by a standard AFM method using colloidal probes. Viscoelasticity parameters were extracted from the experimental data using a standard viscoelasticity model.

\section{Materials and methods}

\subsection{Setup A: magnetic actuation}

Viscoelasticity measurements were performed by magnetically actuating a microprobe and tracking its position as shown in Fig. 1a. A system (Kummer et al. 2010) consisting of eight electromagnets (see Fig. 1b) in a hemispherical configuration was used for the actuation by generating magnetic field gradients that steer the microprobe. Its force calibration, and localization accuracies are described in Kummer et al. (2010). The microprobe is typically a spherical magnetic bead. It is actuated in the horizontal plane, while its apparent weight is compensated by a constant vertical magnetic force. The
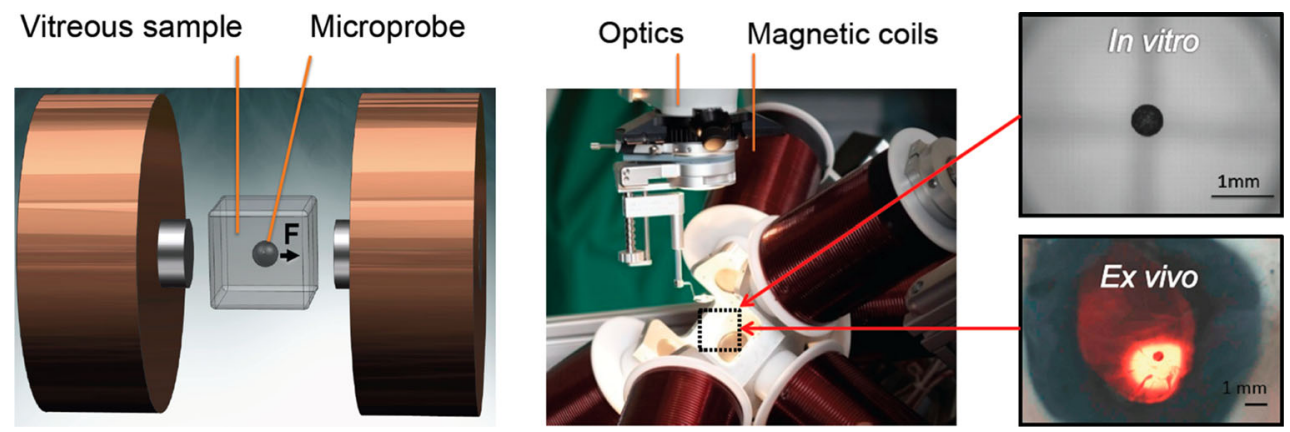

(a) Viscoelasticity measurements by a microprobe.

Fig. 1 a Force $(\mathrm{F})$ exerted on the microprobe in the vitreous is induced by magnetic field gradients. b The fields are generated by eight electromagnets in a hemispherical configuration. The manipulation workspace is located in the middle of the coils (dashed square). An (b) System for wireless magnetic micromanipulation

optical system equipped with a camera images the workspace, where microprobes are used to measure viscoelasticity. Two main types of vitreous models are shown: in vitro artificial vitreous and ex vivo vitreous 
probes in the vitreous samples in vitro (Fig. 1b) and ex vivo (Fig. 1b-2) were tracked using a microscope (Leica M80, Heerbrugg, Germany) equipped with a camera (Gras50S5C-C, Pointgrey, Canada). The imaging system has 1.2$9.2 \mu \mathrm{m} /$ pix resolution for different magnifications. Transscleral illumination through the posterior segment of the eye is applied to achieve high contrast imaging (see Fig. 3). Specifications of Setup A are shown in Table 1. The position of the probe is extracted from the camera frames by background subtraction, binarization, and morphological filtering. Typical frames of real-time tracking of a spherical microprobe in an ex vivo human vitreous are shown in Fig. 2.

\subsection{Setup A: magnetic microprobes}

The probes chosen were sufficiently large to guarantee homogenization of the viscoelastic properties of the vitreous in a location, because the probe sizes were more than an order of magnitude larger than the largest structural components of the vitreous (Bishop 2000). However, the probes are small enough compared to the ocular cavity where the vitreous is located, to provide localized measurements. Hard-magnetic spherical microprobes were used for the ex vivo and in vitro experiments (neodymium iron boron spheres, diameters 0.50 and $0.55 \mathrm{~mm}$, Supermagnete, Gottmadingen, Germany). The remanence of the microprobes was $0.75 \mathrm{~T}$ (characterized by vibration sample magnetometer, MicroMag, Model 3900). For the in vivo rheological experiments the probe needs to fit to a $23 \mathrm{G}$ needle (inner diameter $0.34 \mathrm{~mm}$ with a $0.02 \mathrm{~mm}$ tolerance), because using the needle is a pre-requisite for minimally-invasive sutureless injection into the eyes. Cylindrical cobalt-nickel (CoNi) probes, $1.80 \mathrm{~mm}$ in length and $0.30 \mathrm{~mm}$ in diameter, (Chatzipirpiridis et al. 2015; Fusco et al. 2013) were used in the in vivo experiments. The probes were designed to be narrower than the microspheres in order to fit the probe through a $23 \mathrm{G}$ needle for sutureless injection. However, the length of the probe was increased to generate sufficient magnetic force for manipulation. To enhance biocompatibility (Sivaraman et al. 2013), a polypyrrole coating (3-4 $\mu \mathrm{m})$ was deposited on the probes.

\subsection{Setup B: AFM with a colloidal microprobe cantilever}

The experiments from magnetic microrheology were compared to rheology results obtained using a commercial AFM setup (Bruker, Dimension Edge, Santa Barbara, CA, USA) (Attard 2007). A colloidal probe with a $45-\mu \mathrm{m}$-diameter polystyrene microsphere attached to a silicon cantilever (nominal stiffness $0.03 \mathrm{~N} / \mathrm{m}$, Novascan Technologies, IA, USA) was used in these experiments (see Fig. 4). A relatively soft cantilever and large microsphere were chosen so that the viscoelastic response was driven by the microsphere. The cantilever was immersed in artificial vitreous. The distance from the surface of the sample and from the sample holder to the probe was purposely maintained to be more than $2 \mathrm{~mm}$ to eliminate the effect of wall shear on the hydrodynamics of the probe.

\subsection{Models of the vitreous}

Human donor eyes were provided by the Department of Ophthalmology (Inselspital, University of Bern, Switzerland) after approval by a local ethics committee. The methods adhered to the tenets of the Declaration of Helsinki. The experiments were carried out within $8 \mathrm{~h}$ of death. The eyes (cornea removed for transplantation) were immersed in physiological salt solution and transported in ice-filled packaging to the experimental location. Prior to experiments, the lens and iris were removed to gain direct optical access. Figure 2 shows a probe in the vitreous of a 75-year-old donor used in the creep experiments. Furthermore, manipulation without creep tests was carried in the vitreous of both eyes of 75-, and 76-year-old donors to study mobility: force-displacement behavior was observed similar as in the creep experiments. No signs of previous surgeries were seen in any of the eyes.

Porcine eyes were supplied by a local abattoir in Zurich. They were stored at $5{ }^{\circ} \mathrm{C}$ up to $5 \mathrm{~h}$ prior to use. Each eye was fixed to a custom-made support. Their cornea, lens, and iris were carefully removed to gain direct optical access to the vitreous and to prevent optical artifacts. Microprobe manipulation was carried out with five eyes, however, creep experiments were performed in an eye with a typical displacement response among the eyes.
Fig. 2 a A spherical microprobe tracked while actuated in an ex vivo human vitreous. The square shows the tracked position. b A magnified greyscale image for tracking. The light circular feature below is the optic disk
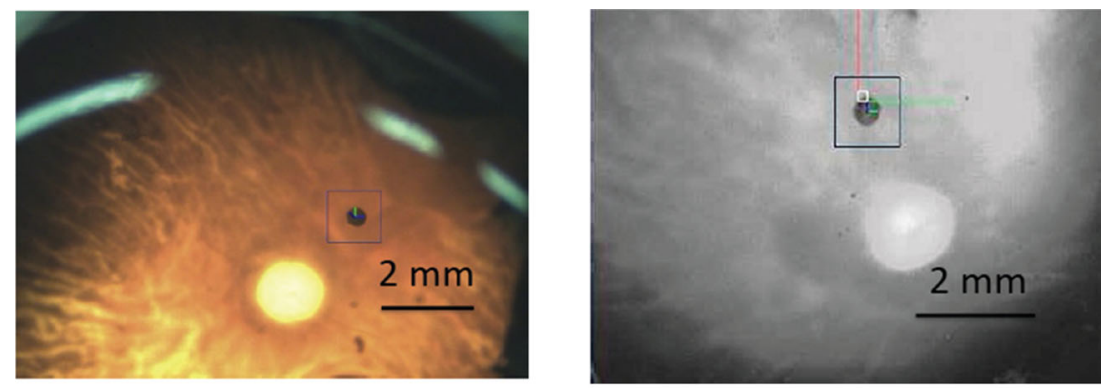

(a)

(b) 
Fig. 3 A $1 \mathrm{~mm}$ long cylindrical microprobe inside the ex vivo human vitreous: a illumination from the side of the optics, and $\mathbf{b}$ transcleral illumination through the posterior segment of the eye

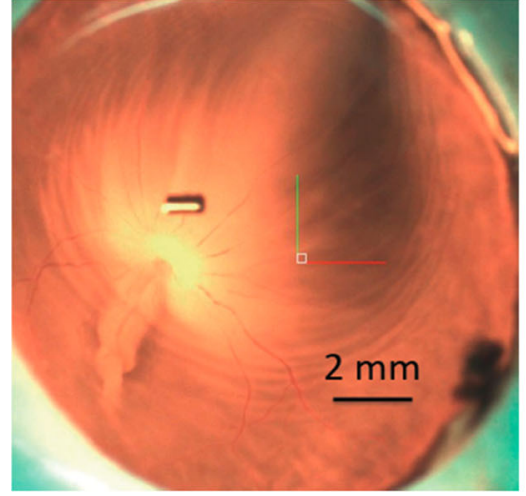

(a)

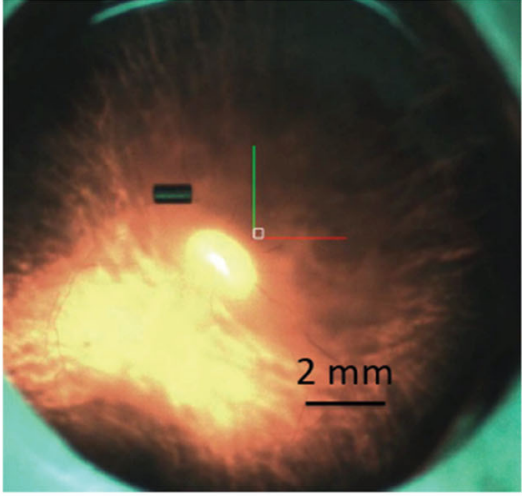

(b)
The experiments with living rabbits were done to prove that the localized viscoelasticity measurements can also be performed in vivo. In vivo experiments were carried out in healthy young New Zealand albino rabbits (Ullrich et al. 2013). The Equine Department of the Vetsuisse Faculty in University of Zurich was in charge of housing and preparations. Prior to experiments a rabbit was anesthetized, muscle paralysis was induced, and its head was supported on Setup A (Fig. 5a) so that the eye of interest was carefully centered. An autoclave sterilized cylindrical $\mathrm{CoNi}$ probe was injected suturelessly into the vitreous using a $23 \mathrm{G}$ needle (Fig. 5b). The eye was imaged to track the probe (see Fig. 5b). No acute medically-relevant biological damage to the eye was found in post mortem examinations based on histology and light microscopy (Fig. 5c). Both eyes of three rabbits were manipulated with forces applied up to $\sim 150 \mu \mathrm{N}$ and the microprobe displacements were up to their body length. Creep measurements from one eye with a typical displacement response are presented. The protocols were performed in accordance with the Swiss Veterinary Office and the ARVO Statement for the Use of Animals in Ophthalmic and Vision Research.

Artificial vitreous was prepared from hyaluronic acid salt (HA salt, Fluka, CAS \# 9067-32-7) and agar (powder form, Fluka, CAS \# 9002-18-0). An aqueous solution of HA and agar was used to model the mechanical behaviour of the HA and collagen in the vitreous (see Fig. 5d-e), respectively (Kummer et al. 2007).

Two types of artificial vitreous were synthesized. The type $1(2.20 \mathrm{mg} / \mathrm{ml} \mathrm{HA}$ salt, $1.10 \mathrm{mg} / \mathrm{ml}$ agar $)$ was prepared to have similar mechanical properties as the porcine vitreous, as in the paper of Kummer et al. (2007). The mechanical properties of the porcine vitreous resemble the ones of the young human vitreous (Lee et al. 1992 and 1994a). The type $2(0.55 \mathrm{mg} / \mathrm{ml}$ HA salt, $0.35 \mathrm{mg} / \mathrm{ml}$ agar) has a concentration of HA which is similar to an elderly human vitreous. The collagen fiber network is more unorganized in an elderly vitreous, hence less affecting to viscoelasticity (Bishop 2000). The agar concentration included in the type 2 mimics the collagen in the elderly human vitreous (Kummer et al. 2007). The HA salt and agar were mixed with DI water $(15 \mathrm{ml})$ and stirred. Subsequently, the container was maintained at $95^{\circ} \mathrm{C}$ (i.e., over agar melting point $85^{\circ} \mathrm{C}$ ) for $20 \mathrm{~min}$ and then mixed vigorously to enhance the homogeneity of the solution.

\subsection{Experimental method}

Viscoelasticity parameters were measured by creep experiments with recently presented methods (Pokki et al. 2012) from in vitro artificial vitreous, and ex vivo vitreous of human and porcine. Creep measurements for viscoelasticity characterization in rabbit vitreous in vivo were demonstrated. The measurements were performed in the central part of the vitreous samples in all experiments. Figure 6a shows data from a typical creep experiment performed by cycling an applied constant force $(\mathrm{F})$ with the following phases: forward $\left(\mathrm{F}=\mathrm{F}_{1}\right.$; at time points $\left.0-t_{1}\right)$, zero-force relaxation $\left(t_{1}-t_{2}\right)$, backward $\left(\mathrm{F}=-\mathrm{F}_{1}\right.$; at time points $\left.\mathrm{t}_{2}-\mathrm{t}_{3}\right)$, and another zero-force relaxation $\left(t_{3}-t_{4}\right)$. The experiment shows the viscoelastic nature of the vitreous. The displacement of a microprobe in response to a constant force has several distinct regions (Fig. 6b) (Lee et al. 1992; Bausch et al. 1998; Sharif-Kashani et al. 2011). An elastic region (the region with time-independent-like force response) is identified right after the force is applied. It typically lasts for $\sim 1 \mathrm{~s}$ and mainly indicates the response of the collagen fiber network in the vitreous (Lai et al. 2008; Sakuma et al. 2004; Sheu et al. 2001; Sharif-Kashani et al. 2011). Subsequently, a retarded elastic region (the nonlinear region following the elastic region) arises from the
Table 1 Specifications of Setup A. In-plane resolution and depth of focus is noted for different magnifications

\begin{tabular}{llll}
\hline Maximum field & Maximum field gradient & In-plane resolution & Depth of focus \\
\hline $50 \mathrm{mT}$ & $1.5 \mathrm{~T} / \mathrm{m}$ & $1.2-9.2 \mu \mathrm{m} / \mathrm{pix}$ & $147-4380 \mu \mathrm{m}$ \\
\hline
\end{tabular}




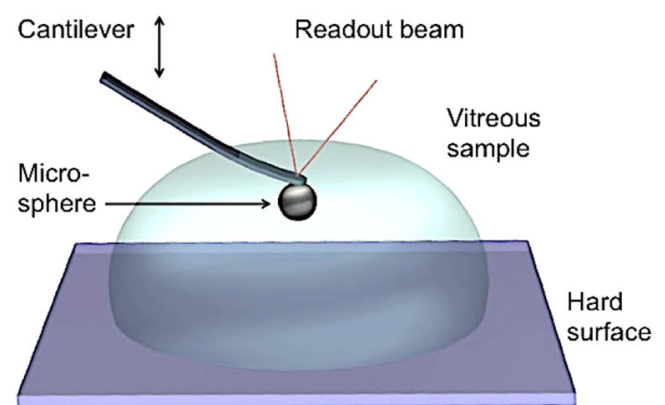

Fig. 4 Setup B based on AFM using a colloidal microprobe cantilever. Its main components are the cantilever that is positioned vertically, and laser/photodiode (readout beam) that detects the cantilever deflection for force measurements. The microprobe (microsphere) attached to the cantilever is immersed to an artificial vitreous sample on a hard glass surface

effects of the responded collagen and hyaluronan (Lee et al. 1992; Sharif-Kashani et al. 2011). Finally, the viscous region (the steady-state linear region) appears after the viscoelastic response of the collagen and hyaluronan (i.e., hyaluronan response in the range of 50-100 s) (Lee et al. 1992; SharifKashani et al. 2011; Almond et al. 1998a, b). After the constant force is removed, the microprobe in the vitreous experiences relaxation (recoil). In this recoil region, the probe returns toward the initial position. The distance between the initial and final positions is known as permanent displacement. To reveal potential hysteresis, a constant force with the same magnitude is subsequently applied in the opposite direction. The microprobe travels through the same regions, and the probe returns close to the initial position. The velocity-response graphs (Fig. 6c-d) also illustrate the regions shown in the position graphs. In the elastic region, the microprobe proceeds at a high velocity (for porcine cadaver eyes $\mathrm{v}_{\max }>1 \mathrm{~mm} / \mathrm{s}$ at $\mathrm{F}_{1}=4.6 \mu \mathrm{N}$ ), and it decelerates in the retarded elastic region. In the viscous region, the velocity decreased asymptotically to $\mathrm{v}_{\text {asym }}$ (for the porcine cadaver eyes $\mathrm{v}_{\text {asym }} \approx 5 \mu \mathrm{m} / \mathrm{s}$ ).

Viscoelasticity parameters of the vitreous were extracted based on the recorded data from creep experiments. Several models have been introduced in the literature to capture the viscoelasticity parameters (Lee et al. 1992; Sharif-Kashani et al. 2011). In order to capture the viscoelastic effects for several tens of seconds of creep experiments, a fourparameter viscoelasticity model based on a Zener model in series with a dashpot was used (Fig. 5e). It is also beneficial in avoiding overfitting, as five-parameter models are typically used for capturing trends of $>100 \mathrm{~s}$ duration creep experiments. This model is solved for the displacement $(p(t))$ in response to a constant force $\left(\mathrm{F}=\mathrm{F}_{1}\right)$ in creep experiments:

$\mathrm{p}(\mathrm{t})=\mathrm{A}^{\prime} \exp (-\mathrm{B} \mathrm{t})+\mathrm{C}^{\prime} \mathrm{t}+\mathrm{D}^{\prime}$.

where $\mathrm{t}$ is time, and $\mathrm{A}^{\prime}, \mathrm{B}, \mathrm{C}^{\prime}$, and $\mathrm{D}^{\prime}$ are model coefficients. To compare the time-dependent displacements for an arbitrary force, creep compliance $\left(\mathrm{J}_{\mathrm{m} / \mathrm{N}}(\mathrm{t})\right.$ in $\left.[\mathrm{m} / \mathrm{N}]\right)$ is derived. The displacement $p(t)$ is normalized by the force $\left(F_{1}\right)$ :

$\mathrm{J}_{\mathrm{m} / \mathrm{N}}(\mathrm{t})=\frac{\mathrm{p}(\mathrm{t})}{\mathrm{F}_{1}}$

To calculate creep compliance $\left(\mathrm{J}_{1 / \mathrm{Pa}}(\mathrm{t})\right.$ in $\left.[1 / \mathrm{Pa}]\right)$ (Ziemann et al. 1994) for an arbitrary size probe, $J_{m / N}(t)$ is scaled by a geometric factor $6 \pi$ and probe radius $\mathrm{R}$ :

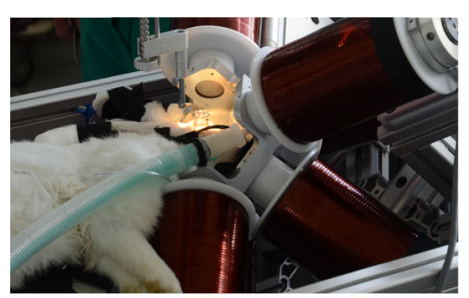

(a) An eye of an anesthetized rabbit centered in the workspace of Setup A.

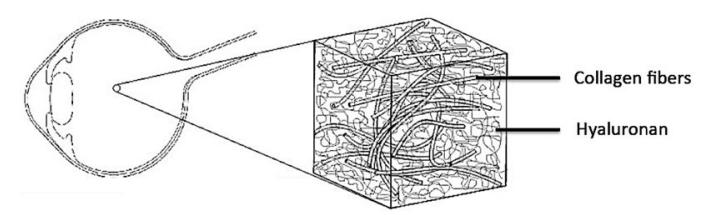

(d) The primary mechanical components of the vitreous.

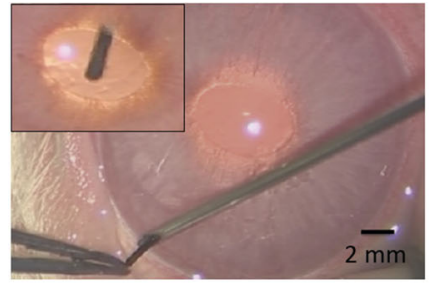

(b) A cylindrical CoNi probe injected nto the vitreous of the rabbit eye.

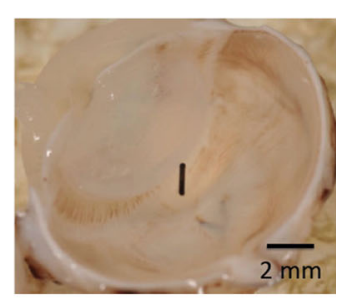

(c) A centered probe between retina and lens post mortem.

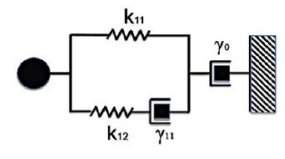

(e) Viscoelasticity model with parameters $\gamma_{0}, \gamma_{11}$, $\mathrm{k}_{11}$, and $\mathrm{k}_{12}$ for mechanical properties.

Fig. 5 Creep measurements are carried out using a probe inserted in the vitreous models in vitro, ex vivo, and in vivo. a The eye of a New Zealand albino rabbit is aligned in the manipulation workspace. b A cylindrical CoNi probe is prepared for an intravitreal injection by a $23 \mathrm{G}$ needle. The inset shows the probe inside the vitreous after a successful injection. $\mathbf{c}$ After the injection, no acute damage in the vitreous was found in post mortem examinations. d The vitreous of the eye has two main components contributing to viscoelasticity, collagen fibers, and hyaluronan. The model in (e) is used to quantify the viscoelastic interaction of a spherical probe in the vitreous. The effect of probe shape can be modelled (Wang et al. 2013) 
Fig. 6 Creep measurements reveal the time-dependent response of a microprobe for a constant force. A typical displacement response of the vitreous is shown in (a)-(b) with the corresponding velocity response in (c)-(d). Remark: the peak velocity $\mathrm{v}_{\max }$ is not shown in (d) as the plot is cropped to show the asymptotically decreasing velocity $\mathrm{v}_{\text {asym }}$

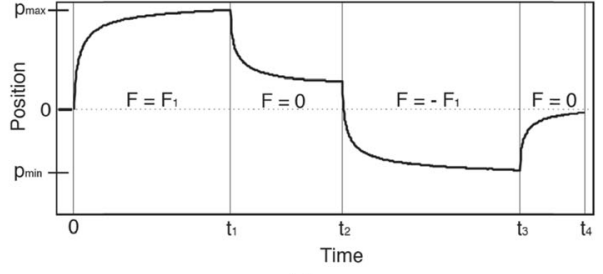

(a)

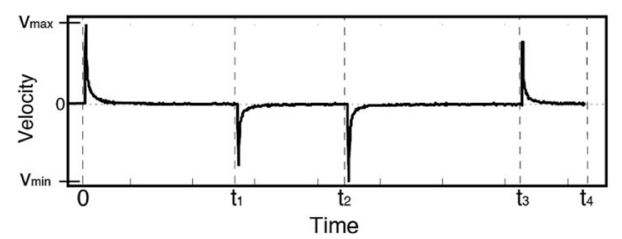

(c)

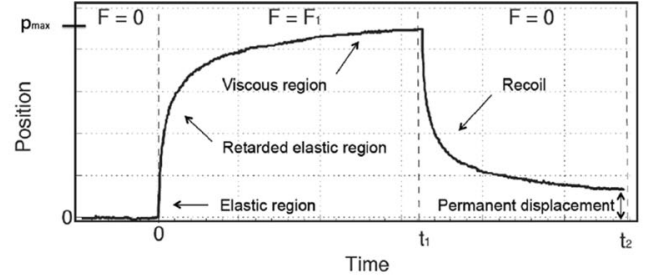

(b)

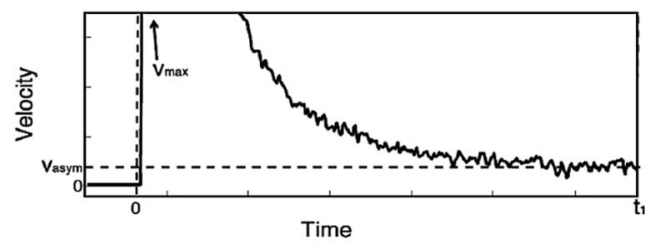

(d)
$\mathrm{J}_{1 / \mathrm{Pa}}(\mathrm{t})=\frac{\mathrm{p}(\mathrm{t})}{\mathrm{F}_{1}} \cdot 6 \pi \cdot \mathrm{R}$

By combining Eq. 1 and Eq. 3, the creep compliance is:

$\mathrm{J}_{1 / \mathrm{Pa}}(\mathrm{t})=\operatorname{Aexp}(-\mathrm{B} \mathrm{t})+\mathrm{C} \mathrm{t}+\mathrm{D}$,

where $\mathrm{A}^{\prime}, \mathrm{C}^{\prime}$, and $\mathrm{D}^{\prime}$ are scaled by $\frac{6 \pi \cdot \mathrm{R}}{\mathrm{F}_{1}}$ to get the coefficients $\mathrm{A}, \mathrm{C}$, and $\mathrm{D}$, respectively. The coefficients A-D are computed from creep experiment data fitted to Eq. 4 using a trust-region-reflective least squares algorithm. The viscoelasticity parameters (steady-state viscosity $\gamma_{0}$ in $[\mathrm{Pa} \cdot \mathrm{s}]$, internal damping $\gamma_{11}$ and elasticity $\mathrm{k}_{1}=\mathrm{k}_{11}+\mathrm{k}_{12}$ in $[\mathrm{Pa}]$ ) were solved from A-D using the following formulae:

$$
\begin{aligned}
& \gamma_{0}=\frac{1}{\mathrm{C}}, \gamma_{11}=\frac{\mathrm{A}}{\mathrm{B} \mathrm{D}^{2}}, \mathrm{k}_{11}=\frac{1}{\mathrm{D}}, \\
& \mathrm{k}_{12}=\frac{\mathrm{A}}{\mathrm{D}^{2}-\mathrm{A} \mathrm{D}}, \text { and } \mathrm{k}_{1}=\frac{1}{\mathrm{D}-\mathrm{A}}
\end{aligned}
$$

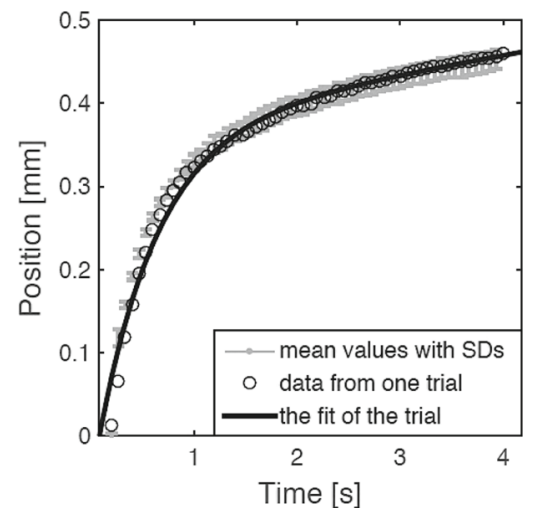

(a) Human ex vivo (age: 75 years).

Fig. 7 Typical examples of displacement in creep experiments with constant forces $F_{1}$. A typical trial is compared to the full data, as mean values with SDs, at a force level $F_{1}$. a Probe displacement data from the ex vivo human vitreous with $\mathrm{F}_{1}=0.46 \mu \mathrm{N}$ (means and SDs are only from
Steady-state viscosity $\left(\gamma_{0}\right)$, and elasticity $\left(\mathrm{k}_{1}\right)$ extracted from the fit of the compliance are calculated. $\gamma_{0}$ and $\mathrm{k}_{1}$ are inversely proportional to a single fitting coefficient and to subtraction of two coefficients, respectively. Hence, they are statistically favoured over internal damping $\left(\gamma_{11}\right)$ that is sensitive to potential variation of three fitting parameters (Eq. 5).

\section{Results and discussion}

\subsection{Setup A: creep experiments in the ex vivo and in vivo vitreous}

Typical examples of creep experiments are presented in Fig. 7. The force level was determined so that the displacement was in the range of a body length of the microprobe. Figure 7 a shows a creep experiment in the ex vivo human vitreous (8 repetitions). Preliminary creep experiments in the in vivo rabbit vitreous (Fig. 7b) were performed to investigate the suitability of the method for in vivo viscoelasticity characterization (5

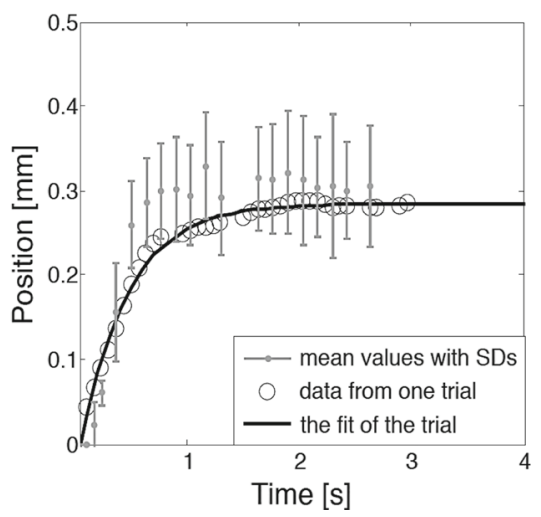

(b) Rabbit in vivo.

the experiments with $\mathrm{F}_{1}=0.46 \mu \mathrm{N} ; 5$ repetitions). The fits to the trials had $\mathrm{R}^{2}>0.984$. b Rabbit in vivo with cylinder off-axis displacement at $0.8 \mathrm{~T} / \mathrm{m}$ while on-axis field $35 \mathrm{mT}\left(\mathrm{F}_{1} \approx 100 \mu \mathrm{N}\right)$. The fits to the trials had $\mathrm{R}^{2}>0.980$ 


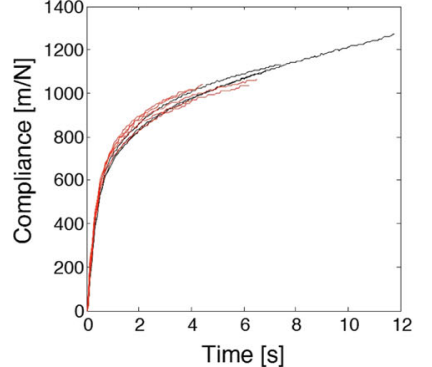

(a) Ex vivo human vitreous measured using two different $\mathrm{F}_{1}$.

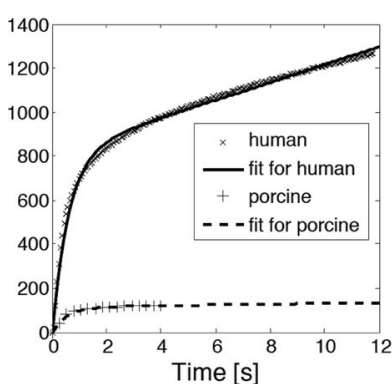

(b) Ex vivo human vitreous versus ex vivo porcine vitreous.

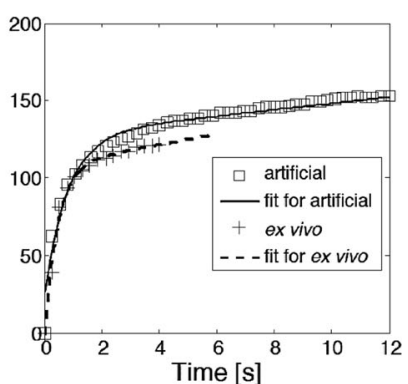

(c) Artificial vitreous type 1 versus ex vivo porcine vitreous.
Fig. 8 Typical examples of creep compliance data. a Separate measurements from the ex vivo human vitreous are shown where two different force levels used: $\mathbf{F}_{1}=0.46 \mu \mathrm{N}$ (red lines), and $\mathbf{F}_{1}=0.91 \mu \mathrm{N}$ (black lines). $\mathbf{b}$ The creep compliances of the ex vivo human vitreous and ex vivo porcine vitreous are presented with their fits $\left(\mathrm{R}^{2}>0.984\right.$ and

repetitions). Figure $7 \mathrm{~b}$ shows displacement in a creep measurement, which indicates a lower compliance compared to ex vivo vitreous samples measured. Apart from species differences this can be due to alterations of the vitreous from in vivo to ex vivo (i.e., elasticity-related storage modulus was found higher in vivo compared to ex vivo in the paper of Zimberlin et al. 2010). Similar force-displacement behaviour was confirmed in the other analogous in vivo rabbit experiments, in both eyes of three rabbits.

Compliance responses to two different force levels in the vitreous of the human donor $\left(\mathrm{F}_{1}=0.46 \mu \mathrm{N}\right.$, and $\left.\mathrm{F}_{1}=0.91 \mu \mathrm{N}\right)$ agree well as presented in Fig. 8a (i.e., requisite for linear viscoelasticity). Figure $8 \mathrm{~b}$ shows the typical difference of the creep compliances between the ex vivo human, and the ex vivo porcine vitreous (5 repetitions). In vitro artificial vitreous (7 repetitions) synthesized based on the mechanical properties of ex vivo porcine vitreous is compared to the ex vivo porcine vitreous in Fig. $8 \mathrm{c} . \mathrm{R}^{2}$ and root-mean-square deviation (RMSD) are noted in the caption as measures of the accuracy of the fits. Viscoelasticity parameters (i.e., elasticity, and steady-state viscosity in Eq. 5) were extracted based on fitting the overall creep data.

The compliances (Fig. 8) and viscoelasticity parameters are consistent with the literature (Lee et al. 1992, 1994a) for

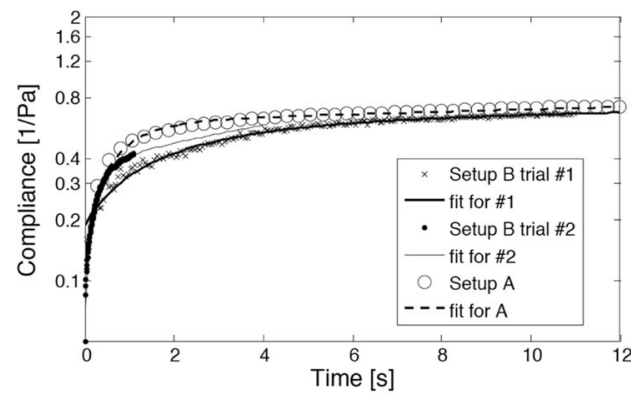

(a)

Fig. 9 a The creep compliance of artificial vitreous type 1 measured using Setup A and Setup B $\left(\mathrm{T}=22-23{ }^{\circ} \mathrm{C}\right)$. Remark: Setup B trial \#3 overlaps with \#2. b Typical creep compliances from Setup B for the
RMSD $<37 \mathrm{~m} / \mathrm{N}, 4 \%$, for all data from human; $\mathrm{R}^{2}>0.976$ and $\mathrm{RMSD}<$ $7.0 \mathrm{~m} / \mathrm{N}, 6 \%$, for all data from porcine). c Creep compliances with their fits from ex vivo porcine vitreous and from artificial vitreous type $1\left(\mathrm{R}^{2}>\right.$ 0.974 and $\mathrm{RMSD}<5.1 \mathrm{~m} / \mathrm{N}, 4 \%$, for all data from artificial vitreous type 1). The experiments were performed at $\mathrm{T}=22-23{ }^{\circ} \mathrm{C}$ except (c)

ex vivo human vitreous (mean $\pm \mathrm{SD}$ values: $\gamma_{0}=3.6 \pm 0.9 \mathrm{~Pa}$. $\mathrm{s} ; \mathrm{k}_{1}=1.6 \pm 0.3 \mathrm{~Pa}$ ) and porcine vitreous (mean \pm SD values: $\gamma_{0}$ $=40.4 \pm 14.5 \mathrm{~Pa} \cdot \mathrm{s} ; \mathrm{k}_{1}=42.6 \pm 39.3 \mathrm{~Pa}$ ). The compliance and viscosity/elasticity of the ex vivo human vitreous varies approximately one order of magnitude from the porcine. Apart from species differences, the increased compliance and reduced viscoelasticity is expected due to the effects of aging in the human vitreous (i.e., collagen network breakdown, liquefaction). The results point out the need of human donor vitreous, or artificial vitreous, in addition to porcine cadaver eyes for studying viscoelastic interaction with the elderly human vitreous. Remark: because the compliance was measured for single locations in the vitreous, the SDs describe uncertainty of a viscosity/elasticity measurement; they do not give a general assessment of homogeneity within the vitreous.

To compare with Setup B, an in vitro model synthesized by defined mechanical properties is used. The synthesized artificial vitreous type 1 has similar creep compliances (Fig. 8c) as ex vivo porcine vitreous. Its viscoelasticity parameters (mean \pm SD values: $\gamma_{0}=96.4 \pm 44.4 \mathrm{~Pa} \cdot \mathrm{s} ; \mathrm{k}_{1}=13.4 \pm 3.9 \mathrm{~Pa}$ ) correspond to the ones of ex vivo porcine vitreous measured. Comparable viscoelasticity parameters in the in vivo rabbit vitreous cannot be directly calculated from the measurements performed using the different probe as there can be a probe-

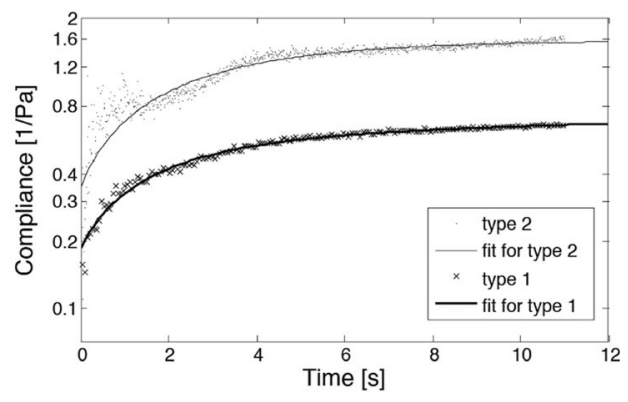

(b)

artificial vitreous type 1 and type 2 are compared to demonstrate that compliances of the artificial vitreous can be tuned for modelling different types of vitreous bodies 
Table 2 Setup A and Setup B compared for elasticity, and steady-state viscosity in artificial vitreous type 1 (mean $\pm \mathrm{SD}$ )

\begin{tabular}{lll}
\hline Method & Elasticity $(\mathrm{Pa})$ & Steady-state viscosity $(\mathrm{Pa} \cdot \mathrm{s})$ \\
\hline Setup A & $13.4 \pm 3.9$ & $96.4 \pm 44.4$ \\
Setup B & $11.5 \pm 5.7$ & $95.6 \pm 84.2$ \\
\hline
\end{tabular}

shape-dependent viscoelasticity effects. However, the parameters can be estimated by modelling the shape effect (Wang et al. 2013).

\subsection{Setup B: artificial vitreous characterized by AFM}

The method using Setup A was traced to standard microrheological measurements by comparing with a validation method using Setup B (see Fig. 9). The creep compliances were scaled against the radius of the probe to obtain the parameters independent of probe size, as described in Eq. 3. Figure 9a shows a typical compliance curve for the artificial vitreous type 1 measured by Setup A (7 repetitions) and by Setup B (3 repetitions). The measured compliances from Setups A and B have similar trends and magnitudes. Based on the fits of the compliances $\left(\mathrm{R}^{2}>0.974\right.$ and $\mathrm{RMSD}<$ $0.024 \mathrm{~Pa}^{-1}$ for Setup A; $\mathrm{R}^{2}>0.988$ and $\mathrm{RMSD}<0.017 \mathrm{~Pa}^{-1}$ for Setup B), the mean values for elasticity and steady-state viscosity were computed (Table 2 ). Mean elasticity and mean steady-state viscosity agree for the two setups. Figure $9 \mathrm{~b}$ shows typical creep curves from AFM characterization of the artificial vitreous type 1 and type 2 . Type 2 was used as an exemplary artificial vitreous to show that adjusting the HA and agar concentrations, the compliance can be tuned. This is important in modelling different types of vitreous bodies. The artificial vitreous type 2 (2 repetitions) has approximately an order of magnitude larger compliance than the artificial vitreous type 1 .

Fig. 10 A cylindrical 1-mmlength microprobe (neodymium iron boron, diameter $0.5 \mathrm{~mm}$ ) was manipulated along a retinal blood vessel in 76-year-old human donor eye vitreous. The frames with the numbers $1-6$ are snapshots during the manipulation, and the trajectory that the probe has moved is marked with dashed arrows. The viscoelastic interaction can be characterized for an arbitrary shape of a microsurgical tool (Wang et al. 2013)

\section{Conclusions}

A minimally-invasive method using magnetic microrheology to characterize the viscoelasticity parameters of vitreous body is described. A new in vivo method for localized viscoelasticity characterization in the vitreous is provided. The measurements were performed using spherical and cylindrical microprobes (see Fig. 10) with the assistance of a magnetic manipulator setup capable of applying magnetic forces wirelessly. The force applied by the manipulator can be controlled within precision described in the paper of Kummer et al. (2010), and the position of the microprobes can be tracked using a customized microscope. Viscoelastic properties of ex vivo human and ex vivo porcine vitreous samples were measured. In addition, minimally-invasive in vivo experiments in an intact eye of a rabbit were performed using the setup described. Independent experiments using a colloidal probe with an AFM setup were performed to verify the results and trace them to microrheology standards. A four-parameter model based on a Zener model in series with a dashpot is used to extract two viscoelasticity parameters, elasticity, and steady-state viscosity.

This method provides a new characterization tool for the vitreous that has the potential for enhanced design of surgical instruments (i.e., shape, size, and coating), diagnosis, and better treatments. Diseases change macromolecular organization within the vitreous that alter spatially-varying viscoelastic properties. Measuring localized viscoelasticity could provide information on diseases (e.g., diabetic vitreo-retinopathy; Fankhauser 2012). To characterize spatially-varying viscoelasticity, this method allows measurements at different locations or using multiple probes at the same time. The fundamental limit for the spatial resolution of the measurement method is the size of the magnetic probe that can be shrunk down to sub-micrometer scales. Quantification of viscoelasticity is required in optimizing the control of ophthalmic instruments used in vitreal manipulation to improve precision,

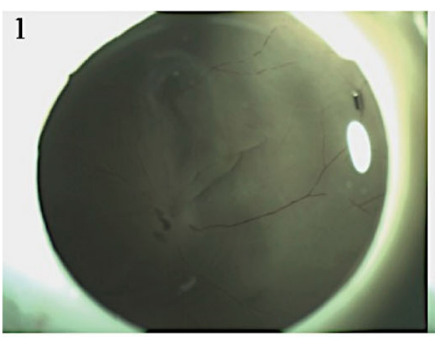

2
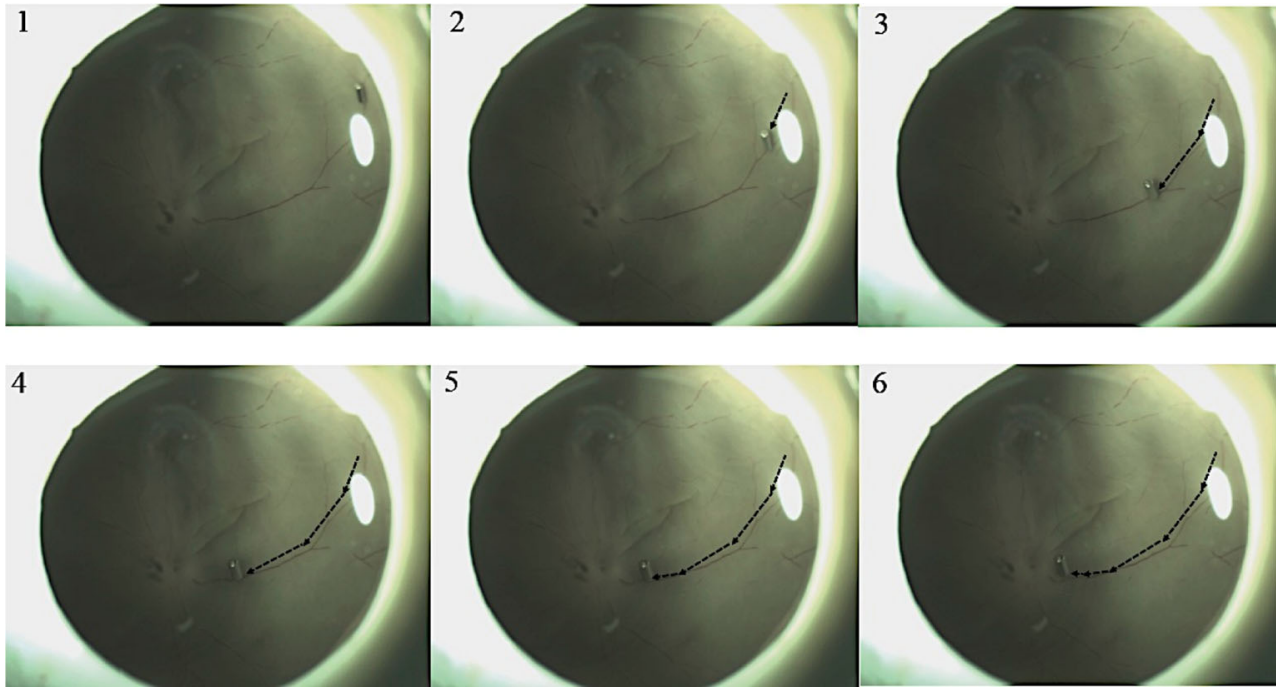

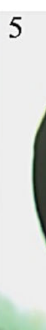

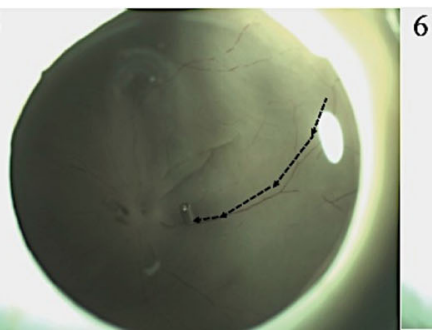

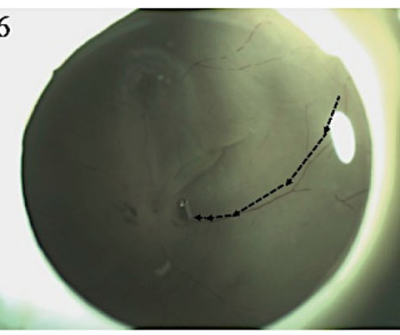


safety, and efficacy. Control parameters of an intraocular microsurgical tool (e.g., Sharif-Kashani et al. 2013; Lee et al. 2013; Lo et al. 2009) or an ophthalmic microrobot (Ullrich et al. 2013; Ergeneman et al. 2012) can be set via an accurate characterization of the viscoelastic interaction with the vitreous. This information can also be used to identify safety limits to minimize risk of vitreo-retinal disorders (e.g., retinal tear happening with $\sim 1 \mathrm{mN}$ forces; He et al. 2013) or interaction with retinal blood vessels (Ergeneman et al. 2011).

Acknowledgments This work was supported by the European Research Council Advanced Grant BOTMED. We would like to acknowledge Christos Bergeles, and George Chatzipirpiridis. Dr. Simon A. Pot and Dr. Bernhard M. Spiess (the Equine Department of the Vetsuisse Faculty in University of Zurich) are acknowledged for their work involving animal housing, surgical/anesthetic procedures, and examinations. Furthermore, we acknowledge Jiaming Yang, Norman Pedrini, Alessandro Schäppi, and Simone Gervasoni for contributing in preparing CAD illustrations.

\section{References}

A. Almond, A. Brass, J.K. Sheehan, J. Mol. Biol. 284, 1425 (1998a)

A. Almond, A. Brass, J.K. Sheehan, Glycobiology 8, 973 (1998b)

R.R. Ansari, K.I. Suh, S. Dunker, N. Kitaya, J. Sebag, Exp. Eye Res. 73, 859 (2001)

P. Attard, J. Phys. Condens. Matter 19, 473201 (2007)

A.R. Bausch, F. Ziemann, A.A. Boulbitch, K. Jacobson, E. Sackmann, Biophys. J. 75, 2038 (1998)

P.N. Bishop, Prog. Retin. Eye Res. 19, 323 (2000)

G. Chatzipirpiridis, O. Ergeneman, J. Pokki, F. Ullrich, S. Fusco, J.A. Ortega, K.M. Sivaraman, B.J. Nelson, S. Pané, Adv. Healthc. Mater. 4, 209 (2015)

O. Ergeneman, J. Pokki, V. Počepcová, H. Hall, J.J. Abbott, B.J. Nelson, J. Med. Devices 5 (2011)

O. Ergeneman, G. Chatzipirpiridis, J. Pokki, M. Marin-Suárez, G.A. Sotiriou, S. Medina-Rodriguez, J.F.F. Sanchez, A. FernandezGutiérrez, S. Pané, B.J. Nelson, IEEE Trans. Biomed. Eng. 59, 3104 (2012)

F. Fankhauser II, Acta Ophthalmol. (Copenh) 90, e173 (2012)

J.D. Ferry, Viscoelastic properties of polymers, 3rd edn. (Wiley, New York, 1980)

B.A. Filas, Q. Zhang, R.J. Okamoto, Y.B. Shui, D.C. Beebe, Invest. Ophthalmol. Vis. Sci. 55, 55 (2014)

S. Fusco, G. Chatzipirpiridis, K. Sivaraman, O. Ergeneman, B.J. Nelson, S. Pané, Adv. Healthc. Mater. 2, 1037 (2013)
X. He, M. Balicki, P. Gehlbach, J. Handa, R. Taylor, I. Iordachita, Conf. Proc. IEEE ICRA (2013)

M.P. Kummer, J.J. Abbott, S. Dinser, B.J. Nelson, Conf. Proc. IEEE Eng. Med. Biol. Soc. (2007)

M.P. Kummer, J.J. Abbott, B.E. Kratochvil, R. Borer, A. Sengul, B.J. Nelson, IEEE Trans. Robot. 26, 1006 (2010)

G. Lai, Y. Li, G. Li, Int. J. Biol. Macromol. 42, 285 (2008)

B. Lee, M. Litt, G. Buchsbaum, Biorheology 29, 521 (1992)

B. Lee, M. Litt, G. Buchsbaum, Biorheology 31, 327 (1994a)

B. Lee, M. Litt, G. Buchsbaum, Biorheology 31, 339 (1994b)

C.Y. Lee, Y.S. You, S.H. Lee, H. Jung, Biomed. Microdevices 15, 841 (2013)

R. Lo, P.Y. Li, S. Saati, R.N. Agrawal, M.S. Humayun, E. Meng, Biomed. Microdevices 11, 959 (2009)

L.I. Los, R.J. van der Worp, M.J. van Luyn, J.M. Hooymans, Invest. Ophthalmol. Vis. Sci. 44, 2828 (2003)

N. Nguyen, J. Sebag, Myopia and related diseases, E. Midena (ed), (Ophthalmic Communications Society, New York, 2005), pp. 137145

J. Pokki, O. Ergeneman, C. Bergeles, H. Torun, B. J. Nelson, Conf. Proc. IEEE Eng. Med. Biol. Soc. (2012)

T.L. Ponsioen, J.M. Hooymans, L.I. Los, Prog. Retin. Eye Res. 29, 580 (2010)

T. Sakuma, Y.Y. Won, J. Sueda, N. Usumoto, D.A. Weitz, T. Hirose, Invest. Ophthalmol. Vis. Sci. 45, 1948 (2004)

E.W. Schneider, M.W. Johnson, Clin. Ophthalmol. 5, 1151 (2011)

J. Sebag, Graefes Arch. Clin. Exp. Ophthalmol. 225, 89 (1987)

J. Sebag, Graefes Arch. Clin. Exp. Ophthalmol. 231, 257 (1993)

J. Sebag, B. Buckingham, M.A. Charles, K. Reiser, Arch. Ophthalmol. 110, 1472 (1992)

J. Sebag, S. Nie, K. Reiser, M.A. Charles, N.T. Yu, Invest. Ophthalmol. Vis. Sci. 35, 2976 (1994)

J. Sebag, R.R. Ansari, S. Dunker, S.I. Suh, Diabetes Technol. Ther. 1, 169 (1999)

J. Sebag, R.R. Ansari, K.I. Suh, Graefes Arch. Clin. Exp. Ophthalmol. 245, 576 (2007)

P. Sharif-Kashani, J. Hubschman, D. Sassoon, H.P. Kavehpour, J. Biomech. 44, 419 (2011)

P. Sharif-Kashani, K. Nishida, H. Pirouz Kavehpour, S.D. Schwartz, J.P. Hubschman, Retina 33, 166 (2013)

M.T. Sheu, J.C. Huang, G.C. Yeh, H.O. Ho, Biomaterials 22, 1713 (2001)

K. Sivaraman, B. Özkale, O. Ergeneman, T. Lühmann, G. Fortunato, A.M. Zeeshan, B.J. Nelson, S. Pané, Adv. Healthc. Mater. 2, 591 (2013)

F. Ullrich, C. Bergeles, J. Pokki, O. Ergeneman, S. Erni, G. Chatzipirpiridis, S. Pané, C. Framme, B.J. Nelson, Invest. Ophthalmol. Vis. Sci. 54, 2853 (2013)

Z. Wang, J. Pokki, O. Ergeneman, B.J. Nelson, S. Hirai, Conf. Proc. IEEE Eng. Med. Biol. Soc. (2013)

F. Ziemann, J. Rädler, E. Sackmann, Biophys. J. 66, 2210 (1994)

J.A. Zimberlin, J.J. McManus, A.J. Crosby, Soft Matter 6, 3632 (2010) 